\begin{tabular}{ccc}
\hline International Journal of Engineering \& Technology, $7(2.19)(2018) 84-86$ \\
SPC & Website $:$ www.sciencepubco.com/index.php/IJET \\
Research paper & Technology \\
\hline
\end{tabular}

\title{
Recognition of Image Verification Using Lossless Predictive Coding
}

\author{
L.Leema Priyadarsini ${ }^{*}$, D.Femi ${ }^{2}$, S.Thylashri ${ }^{3}$, K.Prema ${ }^{4}$ \\ ${ }^{1,2,3,4}$ Assistant Professor \\ Department Of Computer Science And Engineering, School Of Computing, \\ Vel Tech Rangarajan Dr. Sagunthala R\&D Institute Of Science And Technology, \\ Avadi, Chennai-600 062, Tamil Nadu, India. \\ *Lingalogan@Gmail.Com
}

\begin{abstract}
Authentication of image plays a vital role in the end to end communication of file sharing system. In record sharing frameworks, there is a possibility for change of the first information by unapproved arbiters. In the proposed strategy, an exertion is advanced to perceive the vindictive assault in the first picture through prescient lossless coding. To approve the got picture, an encoded prescient quantized picture is utilized. This strategy gives the vital security other than honest to goodness varieties while identifying illicit changes. Lossless prescient coding gives productive encoding by misusing the connection between the projection of the first and got pictures.
\end{abstract}

Keywords: Catchphrases Predictive lossless coding, Predictive RSA encryption and unscrambling.

\section{Introduction}

By and large, in the document sharing frameworks, picture confirmation procedure pays an imperative part to guarantee the innovation of the picture. It ought to be noticed that untrusted gobetweens may alter the first information by meddling with the conveyance of specific records, or piggybacking unauthentic substance. Recognizing true blue encoding forms from vindictively altered ones is essential in applications that convey media conten through untrusted delegates. The issue is additionally testing if some real changes, for example, editing and resizing a picture, are permitted not withstanding lossy compression. Extra alterations won't not change the significance of the substance, but rather could be misclassified as altering. Client may likewise be keen on limiting altered locales. Recognizing real encodings with conceivable modifications from altering and limiting altering are the difficulties tended to. We apply farsighted lossless coding and quantifiable methods to deal with the photo approval issue. Past procedures for picture affirmation fall into four social affairs: wrongdoing scene examination, watermarking, solid hashing and appropriated source coding. In modernized wrongdoing scene examination, the customer affirms the believability of a photo only by checking the got content [1-2]. Heartbreakingly, without any information from the initial, one can't thoroughly certify the genuineness of the got content since content arbitrary to the first may clear criminological checking. Second option for picture approval is watermarking. A semi-delicate watermark is embedded into the host flag waveform without perceptual bending. Customers can assert validity by removing the watermark from the got content [35]. Sadly, without any information from the initial, one can't thoroughly avow the trustworthiness of the got content since content arbitrary to the first may clear criminological checking. Second option for picture approval is watermarking. A semi-delicate watermark is embedded into the host flag waveform without perceptual distortion. Customers can assert validity by removing the watermark from the got content [3-5]. Shockingly, watermarking confirmation isn't backward great with in advance encoded substance. Another approach is intense hashing, which is animated by cryptographic hashing. In this framework, the customer checks the respectability of the got content using a little measure of data got from the main substance [6]. The essential drawback in overwhelming hashing is even little change in the hash regard i.e., due to weight moreover appear as adjusted picture. Besides, the other approach in picture affirmation is coursed source coding. In the coursed source coding methodology, the measure of the checked data is precisely picked and this information empowers us to perceive good 'ol fashioned encoding assortments of the photo and silly alterations [4]. The inconvenience in appropriated source coding is, picture projection and Slepian-wolf coder causes some adversity in data as a result of weight. In order to vanquish each one of these burdens in this paper we propose a framework known as lossless exactness coding which makes the resultant data without any hardships i.e., the data is changed over into adaptable twofold configuration. Thusly we can achieve pressure high proportion and high PSNR.

\section{Image Verification Technique}

The user makes the verification based on the end image and the verification data. The legal and the tampered channels are the two channels present in the verification system. In the legal channel there is no manipulation of the image instead the end image will 
be in compressed format. In the tampered channel image will have some manipulations in addition to compression.

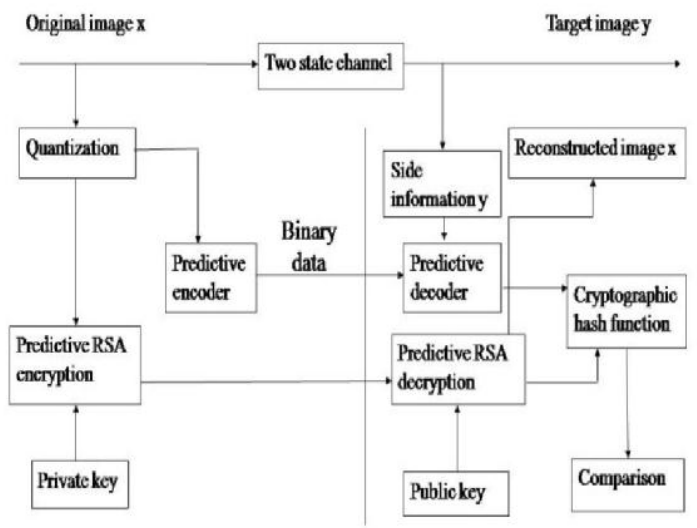

Fig 1 Image verification

Figure 1 demonstrates the picture confirmation framework through lossless prescient coding. In this strategy for prescient coding, the first picture $\mathrm{x}$ is quantized and the subsequent information is sent to prescient encoder and furthermore for prescient encryption. The quantized picture which is sent to the encoder experiences isolate change and is then changed over into double information. This paired information is exchanged to the decoder where it is utilized to re-build the first picture $\mathrm{x}$. Amid encryption the picture is confirmed by utilizing private key where as the picture is decoded by utilizing open key. This checked picture goes about as recreated picture $\mathrm{x}$. The check choice depends on the objective picture y and reproduced picture $\mathrm{x}$. Performing hash work for both decoder and unscrambling picture looking at both the outcome, the recipient perceives that picture $y$ is altered.

\section{Encoder}

The info picture is perused as JPEG document, which is then changed over into a dim scale picture as appeared in Fig. 2. The dim picture is then subdivided into sub hinders; the discrete change is connected to all the sub squares. By utilizing edge the picture is changed over into twofold information as appeared in Fig. 3. The paired information is then exchanged to the end zone.

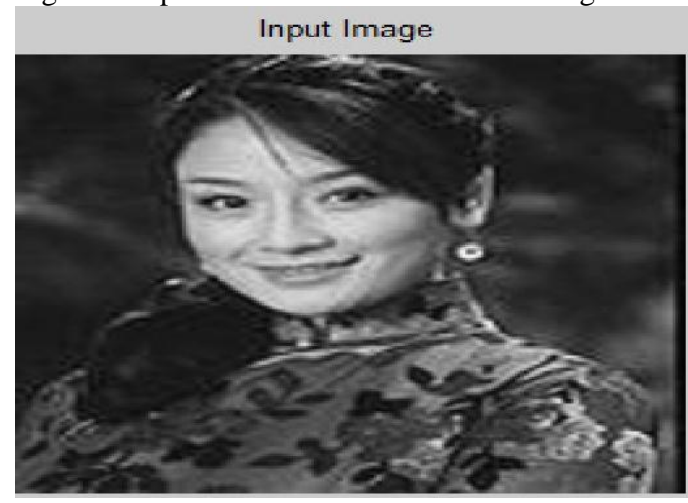

Fig 2 Input image

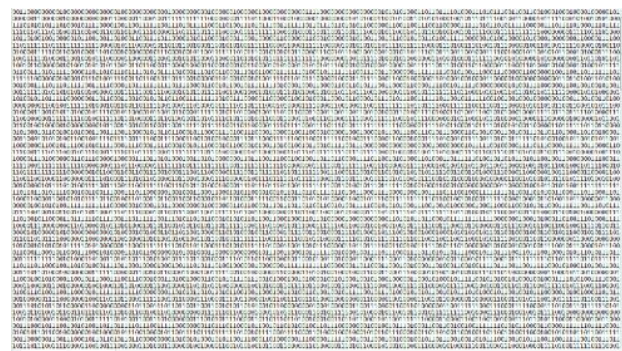

Fig 3 Encoded binary data

\section{Encryption}

The dim scale picture gets quantized and exchanged to the end territory utilizing private key amid encryption. The subsequent picture will be more lawful when contrasted with the picture exchanged utilizing encoder. Figure 6 demonstrates the private key utilized for encryption and Fig. 5 demonstrates the twofold information of encryption.

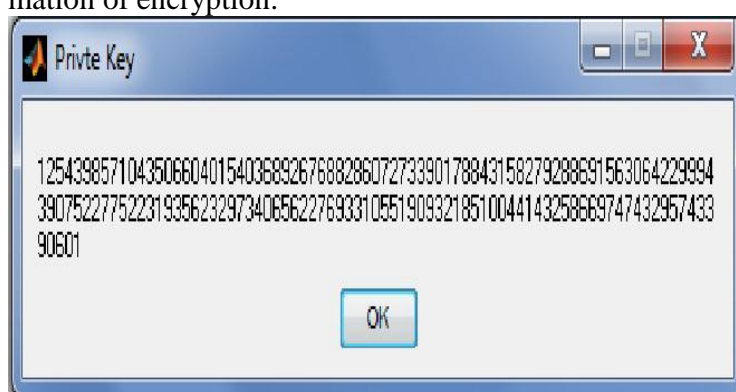

Fig 4 Private key

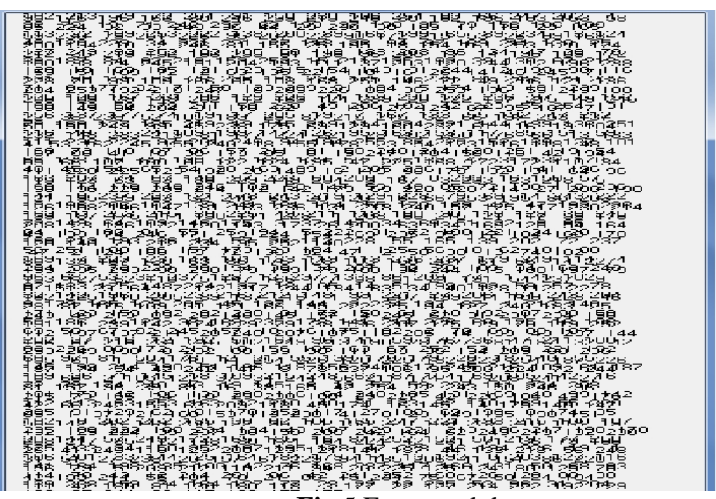

Fig 5 Encrypted data

\section{Decoder}

The encoded picture is then recovered by the beneficiary which is later decoded utilizing decoder to acquire a yield picture. The double information got by the decoder changes over the packed picture into content configuration and it is then decoded to deliver a unique picture as appeared in Fig. 6.

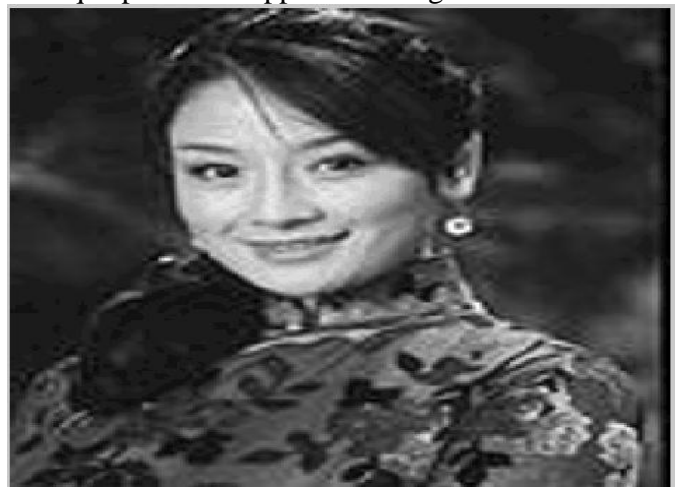

Fig 6 Decoded Image

\section{Decryption}

The encoded twofold information which is gotten by the beneficiary is then decoded by utilizing open key unscrambling. The twofold information is decoded and it reshapes the single dimensional information in to two measurements. Figure 7 demonstrates people in general key and Fig. 8 demonstrates the yield of unscrambling. 


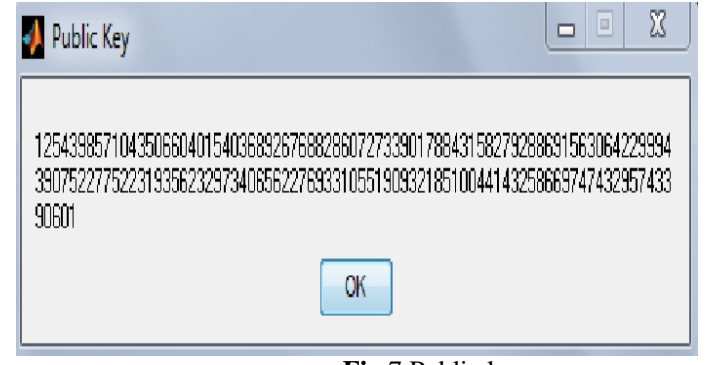

Fig 7 Public key

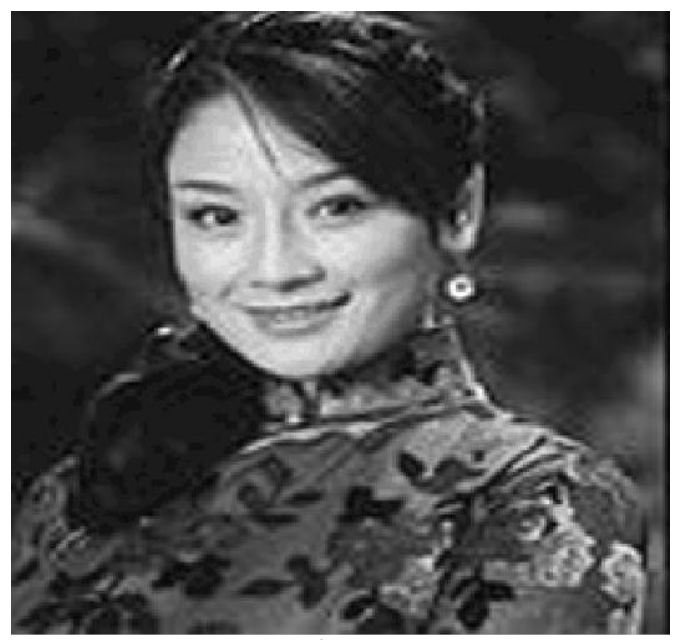

Fig 8 Decrypted Image

\section{Performance}

The execution characters, for example, top flag to clamor proportion (PSNR), pressure proportion of the current strategy is contrasted and the proposed technique.

\subsection{Psnr}

Pinnacle flag to clamor proportion must be high so as to acquire a fantastic picture. In proposed technique PSNR esteem is high contrasted with the current one as appeared in Fig9

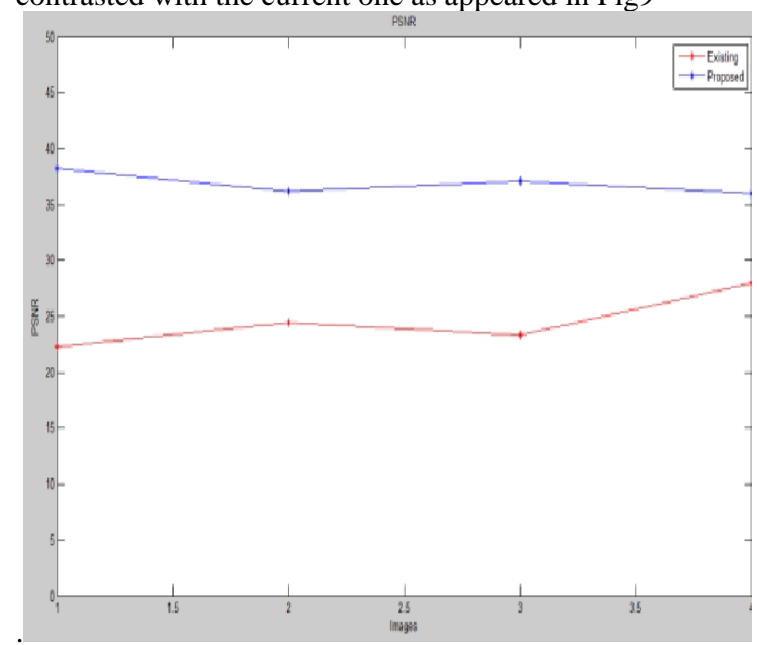

Fig 9 PSNR and image

\subsection{Compression Ratio}

The pressure proportion ought to be high for astounding pictures. The pressure proportion for proposed strategy is moderately high contrasted with the current method as appeared in Fig. 10.

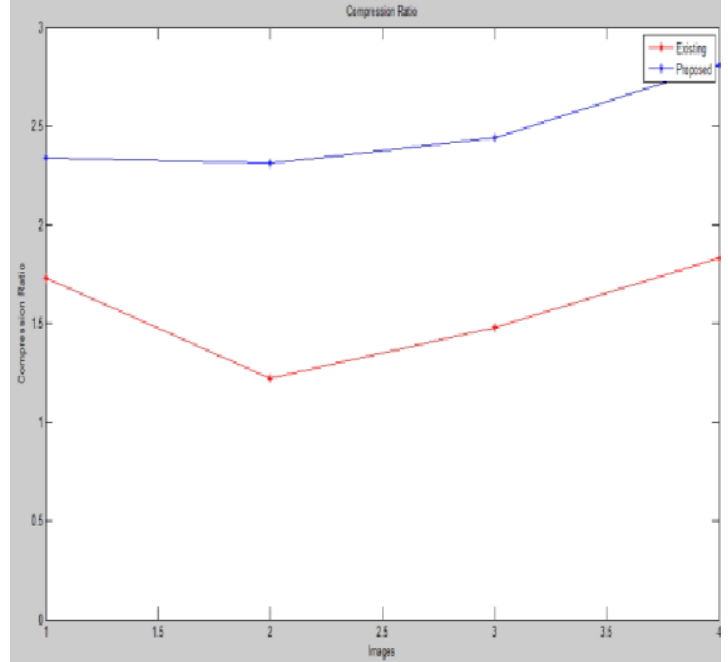

Fig 10 compression ratio and image

\section{Conclusion}

In the proposed strategy, an exertion is made to utilize lossless prescient coding for picture confirmation issue. In the document sharing procedure, there are two potential outcomes for the first substance to get adjusted. They are (I) because of pressure of record amid exchanging and (ii) by unapproved arbiters. So it is critical to track the kind of assault on the record content. With a specific end goal to approve the got picture, encoded picture is utilized. This method gives most extreme security against honest to goodness varieties while recognizing unlawful varieties. The execution parameters, for example, the flag to-clamor proportion and pressure proportion are observed to be better in lossless prescient coding.

\section{References}

[1]. Javier Molina-Garcia, Rogelio Reyes-Reyes, Volodymyr Ponomaryov, Clara Cruz-Ramos,'Watermarking Algorithm for Authentication and Self-Recovery of Tampered Images Using DW", IEEE 2016.

[2]. S. Amir Hossein Tabatabaei, Obaid Ur-Rehman, Natasa Zivic and Christoph Ruland,"Secure and robust two phase image authentication", 1520-9210 (c) 2015 IEEE

[3]. G. Nian, X. Tang, D. Wang, H. Liu, "Print-scan resilient data hiding scheme applied in certificate verification", Proc. CISP, 2010.

[4]. S. Tachaphetpiboon, K. Thongkor, T. Amornraksa, E.J. Delp, "Digital watermarking for color images in hue-saturation-value color space", Journal of Electronic Imaging, vol. 23, no. 3, 2014.

[5]. P. Bas, J.M. Chassery, B. Macq, "Geometrically invariant watermarking using feature points", IEEE Trans. Image Process., vol. 11, no. 9, pp. 1014-1028, 2002.

[6]. A. Bovik, "The Essential Guide to Image Processing" in Elsevier Inc., USA:, 2009. 